\title{
Blood Pharmacokinetics Imaging by Noninvasive Heart Fluorescence Tomography and Application to Kidney Glomerular Filtration Rate Assessment $\$$ \$
}

\author{
Bagna Bao, Kristine O. Vasquez, Guojie Ho, Jun Zhang, Jeannine Delaney, \\ Milind Rajopadhye, and Jeffrey D. Peterson
}

PerkinElmer Inc., Hopkinton, Massachusetts

Received January 31, 2019; accepted May 24, 2019

\begin{abstract}
In mouse pharmacokinetic (PK) studies, current standard methods often require large numbers of animals to support collection of blood samples serially over a defined time range. We have developed and validated a noninvasive fluorescence molecular tomography (FMT) heart imaging approach for blood PK quantification that uses small numbers of mice and has the advantage of repeated, longitudinal live imaging. This method was validated using a variety of near infrared (NIR) fluorescent-labeled molecules, ranging in size from 1.3 to $150 \mathrm{kDa}$, that were assessed by microplate blood assays as well as by noninvasive FMT 4000 imaging. Excellent agreement in kinetic profiles and calculated PK metrics was seen for the two methods, establishing the robustness of this noninvasive optical imaging approach. FMT heart
\end{abstract}

imaging was further assessed in the challenging application of inulin-based glomerular filtration rate (GFR) measurement. After a single bolus injection of an NIR fluorescent-labeled inulin probe in small cohorts of mice ( $n=5$ per group), 2-minute heart scans (at $2,6,15,30$, and 45 minutes) were performed by FMT imaging. GFR was calculated using two-compartment PK modeling, determining an average rate of $240 \pm 21 \mu \mathrm{l} / \mathrm{min}$ in normal mice, in agreement with published mouse GFR ranges. Validation of GFR assessment in unilaterally nephrectomized mice and cyclosporin A-treated mice both measured $~ 50 \%$ decreases in GFR. Imaging results correlated well with ex vivo plasma microplate assays for inulin blood kinetics, and the decreases in GFR were accompanied by increases in plasma creatinine and blood urea nitrogen.

\section{Introduction}

The optimization and characterization of small molecule drugs and large biomolecule therapeutics (or "biologics") is a multifaceted process requiring the generation of extensive in vitro and in vivo research data sets. Characterizing agent blood pharmacokinetics (PK) and exposure profiles in animals is an important part of this process, providing guidance for formulation, optimizing bioavailability and clearance, establishing dosing regimens, determining the projected human dose (Toon, 1996), and understanding the $\mathrm{PK} /$ pharmacodynamic $(\mathrm{PD})$ relationship.

In early drug discovery, preclinical PK studies are often performed in mice for a direct comparison of PK and PD responses in mouse efficacy models. Unfortunately, the small body size of mice limits the amounts of blood available for analytical measurements of drug and PD marker levels. This is particularly an issue for large molecule biologics, which often require collection of large blood samples for ligandbinding immunoassays, large cohorts of mice, larger amounts of drug material, and more labor resources. As a result, PK

https://doi.org/10.1124/jpet.119.257071.

S This article has supplemental material available at jpet.aspetjournals.org. studies in mice are typically low-throughput and high-resource efforts. In 2011, we used fluorescence molecular tomography (FMT) imaging to measure whole-body three-dimensional (3D) imaging biodistribution in mice (Vasquez et al., 2011) and discovered that the longitudinal heart data sets agreed well with more labor-intensive microplate plasma assay results. One obvious advantage of the FMT heart PK approach is the ability to acquire full kinetic blood curves from individual mice through longitudinal live imaging, an approach that generally needs only three to five injected mice. There are certainly other nonimaging blood microsampling approaches (e.g., NanoDrop and liquid chromatographytandem mass spectrometry analysis of dried blood spots) that require collection of only small blood samples with few mice (Zhang et al., 2016). However, such approaches may still require extensive sample manipulation and analytical runs, whereas FMT heart imaging requires no sample collection and only minimal analysis for near real-time results. The mice can also be retained for weeks to repeat GFR assessment for longitudinal kidney toxicity studies. This is a potentially major benefit both logistically and ethically, supporting current worldwide initiatives in the 3Rs (reduce, refine, and replace) of animal welfare (Balls, 2015).

To further validate FMT heart imaging for PK studies, we used a variety of near infrared (NIR) fluorescent-labeled

ABBREVIATIONS: 3D, three-dimensional; AS680, AngioSense 680EX; AS750, AngioSense 750EX; AUC, area under the curve; BUN, blood urea nitrogen; CsA, cyclosporin A; FMT, fluorescence molecular tomography; FRS680, FolateRSense 680; GFR, glomerular filtration rate; GFR680, GFRVivo 680; IS750, IntegriSense 750; NIR, near infrared; PD, pharmacodynamics; PET, positron emission tomography; PK, pharmacokinetics; ROI, region of interest; $t_{1 / 2}$, half-life; UNX, unilaterally nephrectomized; VT680XL, VivoTag 680XL. 
agents, ranging in molecular mass from 1.3 to $150 \mathrm{kDa}$. A careful comparison of 1.6-kDa FolateRSense 680 (FRS680) blood kinetics by FMT imaging ( $n=3$ mice) and by serial bleeding ( $n=24$ mice) showed a high correlation between results. In addition, multiple PK parameters derived from three-compartment modeling, including half-life $\left(t_{1 / 2 \alpha}, t_{1 / 2 \beta}\right.$, $\left.t_{1 / 2} \gamma\right)$, volume of distribution, clearance rates, and area under the curve (AUC), were in excellent overall agreement for both methods. Four additional NIR fluorescent-labeled molecules (a small molecule integrin antagonist, two large vascular agents, and labeled IgG1) were also assessed by FMT heart imaging in comparison with serial bleeds, confirming that FMT heart imaging is effective and accurate for determining PK profiles.

The FMT heart imaging technique can work for both small molecule and large molecule PK assessment; however, the best applications of this technique are 1) assessing the PK of large molecule biologics in which the size is not significantly changed by the addition of the fluorescent label (e.g., IgGs and large therapeutic proteins) and 2) assessing the PK of NIR fluorescent conjugates of any size (e.g., for imaging probe characterization, blood kinetic applications, and developing drug-delivery conjugates). For the second type of approach, we identified a unique probe/application that can benefit from a noninvasive heart tomography approach: glomerular filtration rate (GFR) measurement using 6-kDa NIR fluorescent-labeled inulin, GFR-Vivo 680 (GFR680). The rate of disappearance of inulin from the blood is directly proportional to GFR, and changes in GFR can indicate kidney dysfunction due to disease or drug-induced injury. After an intravenous bolus of NIR fluorescent-inulin in hairless SKH-1E mice, longitudinal FMT heart quantification was used to determine blood clearance rates using a two-compartment curve fit, yielding average rates of $240 \pm 21 \mu \mathrm{l} / \mathrm{min}$ in normal mice, which is in close agreement with published GFR data (Qi et al., 2004, 2005; Bivona et al., 2011; Rieg, 2013). Removal of one kidney or treatment of mice with cyclosporin A (CsA) both yielded predictable and quantitative delays in GFR of $\sim 50 \%$. These studies showed accompanying increases in plasma creatinine and blood urea nitrogen (BUN), although as expected they did not correlate well with the magnitude of GFR changes. GFR680, in combination with FMT heart imaging, provides a noninvasive fluorescent imaging approach to generate consistent GFR measurements in models of kidney disease, dysfunction, and drug-induced injury.

\section{Materials and Methods}

Ethics Statement. All experiments were performed in accordance with PerkinElmer guidelines based on recommendations in the National Institutes of Health Guide for the Care and Use of Laboratory Animals. The study protocol was approved by PerkinElmer's In Vivo Imaging Division in accordance with Institutional Animal Care and Use Committee guidelines for animal care and use. Uninephrectomized mice received appropriate surgical procedures at Charles River (Wilmington, MA) 3 days prior to shipping to PerkinElmer for use on day 7. All imaging activities were performed under appropriate anesthesia to minimize animal distress, and no invasive or surgical procedures were used at PerkinElmer in these imaging studies.

Experimental Animals. For PK studies, specific pathogen-free BALB/c, SKH-1E, and uninephrectomized SKH-1E female mice (6-8 weeks of age) were obtained from Charles River, housed five per cage with nesting material, and maintained in a controlled environment $\left(72^{\circ} \mathrm{F}\right.$; 12-hour/12-hour light/dark cycle) under specific pathogen-free conditions with water and low fluorescence chow (Envigo, Cambridgeshire, UK) provided ad libitum.

Fluorescent Probes and Protein Conjugates. Commercially available NIR imaging probes and custom conjugates (PerkinElmer Inc., Waltham, MA) were used to optimize and perform PK studies (summarized in Table 1). All probes were dosed at $2 \mathrm{nmol} / \mathrm{mouse}$ (by fluorescence) intravenously according to the manufacturer's instructions. FRS680 (catalog no. NEV10040EX) is a folate-based imaging probe that detects folate receptor expression. AngioSense 680EX and 750EX (AS680 and AS750; catalog nos. NEV10054EX and NEV10011EX, respectively) are vascular imaging probes. IntegriSense 750 (IS750; catalog no. NEV10873) detects regions of upregulated $\alpha \mathrm{V} \beta 3$ integrin expression associated with cancer, angiogenesis, and other indications. GFR680 (catalog no. NEV30000) is an NIR fluorescentlabeled inulin molecule used to assess GFR calculated from blood clearance kinetics. VivoTag 680XL (VT680XL; catalog no. NEV11119) is an NIR fluorophore that can be used to label large molecule therapeutic proteins and antibodies for biodistribution and PK assessment.

A custom IgG1-fluorophore conjugate was produced using murine IgG1 purchased from Rockland (Gilbertsville, PA) to provide a reference agent for PK studies. $N$-hydroxysuccinimide ester labeling of proteins was performed using a 680-nm fluorophore (VT680XL). Briefly, the $N$-hydroxysuccinimide ester moiety of VT680XL reacts with primary amino groups of proteins to form an amide bond $\mathrm{pH} 7$ for 1 hour at room temperature). In this manner, a small number of lysine residues (generally one to two) for antibodies and other proteins can be labeled with fluorophore. Free fluorophore is removed by size exclusion chromatography. Conjugation ratios were easily assessed by measuring absorbance of the conjugates at 280 and $680 \mathrm{~nm}$ with appropriate extinction coefficients to determine protein and fluorophore concentration, respectively. Labeling for IgG1 (IgG1VT680XL) achieved approximately two fluorophores per protein molecule, and this conjugate was dosed in animals at $2 \mathrm{nmol}$ fluorescence/mouse intravenously. This conjugate showed excellent stability when stored at $4^{\circ} \mathrm{C}$ and, upon in vivo injection, showed no evidence of unconjugated free fluorophore.

In Vivo PK Imaging and Blood PK. Fluorescence imaging requires removal of hair from experimental animals for optimal results. Therefore, prior to the imaging, BALB/c mice were anesthetized with isoflurane gas and hair was removed using depilatory cream (Nair; Church \& Dwight Co., Ewing, NJ). Briefly, the cream was applied two to three times (4 to 5 minutes each time) with gentle rinsing in warm water. Hairless SKH1-E mice, or depilated BALB/c mice, were injected intravenously with $2 \mathrm{nmol} \mathrm{NIR} \mathrm{fluorescent} \mathrm{probes/}$ conjugates and imaged at five to eight selected time points after injection, the first time being within the first 5 minutes for standard PK studies. For the specific application of GFR assessment, precise timing was critical (with the first image acquisition initiated precisely at 1 minute after GFR680 injection) because of the rapid clearance of this probe. At the time of imaging, mice were anesthetized by inhalation in a chamber containing a mixture of isoflurane and oxygen and then imaged using the FMT 4000 in vivo imaging system (PerkinElmer Inc.). For heart region imaging, the anesthetized mice were placed in the supine position, centrally in the imaging cassette, to capture the thoracic region of the animal within the imaging scan field (30-40 scan points, $\sim 2$-minute scan). After positioning the mouse, the imaging cassette was adjusted to the proper depth to gently restrain the mouse and then inserted into the heated docking system (regulated at $\sim 37^{\circ} \mathrm{C}$ ) in the FMT imaging chamber. An appropriate NIR laser diode transilluminated each mouse's body (i.e., passed light through the body of the animal to be collected on the opposite side), with signal detection occurring via a thermoelectrically cooled charge-coupled device camera placed on the opposite side of the imaged animal. Appropriate optical filters allowed the collection of both fluorescence and excitation data sets, and the multiple sourcedetector fluorescence projections were normalized to the paired collection of laser excitation data. 
TABLE 1

Probes and custom conjugates used to optimize and perform PK studies

\begin{tabular}{|c|c|c|c|c|}
\hline Probe & $\begin{array}{l}\text { Molecular } \\
\text { Mass }\end{array}$ & Description & $\begin{array}{l}\text { Excitation/ } \\
\text { Emission }\end{array}$ & Applications \\
\hline & $k D a$ & & $n m$ & \\
\hline FRS680 & 1.6 & Folate-based NIR fluorescent imaging probe & $670 / 690$ & Oncology and inflammation \\
\hline AS680 & 70 & $\begin{array}{l}\text { NIR fluorescent-labeled PEG-polylysine copolymer } \\
\text { used as a vascular imaging probe }\end{array}$ & $670 / 690$ & $\begin{array}{l}\text { Vascularity and vascular leak changes in } \\
\text { oncology and inflammation }\end{array}$ \\
\hline AS750 & 70 & $\begin{array}{l}\text { NIR fluorescent-labeled PEG-polylysine copolymer } \\
\text { used as a vascular imaging probe }\end{array}$ & $750 / 770$ & $\begin{array}{l}\text { Vascularity and vascular leak changes in } \\
\text { oncology and inflammation }\end{array}$ \\
\hline IS750 & 1.28 & $\begin{array}{l}\text { NIR fluorescent-labeled small molecule } \alpha \mathrm{V} \beta 3 \text { integrin } \\
\text { antagonist }\end{array}$ & $755 / 775$ & Oncology, atherosclerosis, angiogenesis \\
\hline GFR680 & $\sim 6.0$ & NIR fluorescent-labeled inulin & $670 / 690$ & Measuring GFR by blood PK \\
\hline $\begin{array}{l}\text { IgG1- } \\
\text { VT680XL }\end{array}$ & 150 & Normal mouse IgG1 labeled with VivoTag 680XL & $665 / 688$ & Control \\
\hline
\end{tabular}

PEG, polyethylene glycol.

Separate nonimaged cohorts of animals were bled for microplate fluorescence assay and quantification of the amount of fluorescentlabeled conjugates. Cohorts were used for terminal blood collection at five to eight selected times to align with imaging data. Briefly, mice were anesthetized using isoflurane gas, and blood was collected through the retro-orbital plexus using glass capillary tubes. Blood was collected into EDTA- or heparin-coated blood collecting tubes for generation of plasma samples. Control plasma was collected to generate a fluorescent standard curve for comparison with collected samples from experimental mice. The standard curve samples and blood collection samples were also diluted 1:2 in DMSO, which ensured the maximal capture of fluorescence despite the presence of proteins that could potentially alter fluorescent signal.

FMT Reconstruction and Analysis. The collected fluorescence data were reconstructed by FMT 4000 system software (TrueQuant 4.0; PerkinElmer Inc.), which compensates for the effects of tissue heterogeneity on light scattering (Mohajerani et al., 2009), allowing for the quantification and $3 \mathrm{D}$ visualization of fluorescence signal within the heart. Three-dimensional regions of interest (ROIs) were drawn to encompass the heart signal within the thoracic region. The total amount of heart fluorescence (in picomoles) was automatically calculated relative to prior calibration generated with known concentrations of appropriate NIR dyes. To prevent loss of low-level fluorescence after dissemination of agents into tissues, no thresholding of fluorescence data sets was applied.

PK Analysis. To determine the blood PK profile of a fluorescent conjugate in living mice, unthresholded heart tomography quantification by TrueQuant software was used. To avoid partial volume effects of fluorescence tomography (i.e., some enlargement of target size due to reconstruction voxel size), slightly oversized ROIs were placed to capture the total fluorescence per heart (in picomoles). These data were converted to nanomoles based on known physiologic parameters; a 25-g mouse has an average of $1.5 \mathrm{ml}$ of blood, $7 \%$ of which (or $\sim 100 \mu \mathrm{l}$ ) is in the heart. All data points in picomoles were divided by $0.1 \mathrm{ml}$, to yield nanomolar results for each time point. A dose of 2000 pmol yields a theoretical time zero blood concentration of 1330 nM (i.e., a time zero heart measurement of $\sim 133$ total pmol). However, fluorescence quantification could be affected by how well the imaging system is calibrated as well as by the fluorescence behavior of the conjugate itself (i.e., how much the fluorescent signal is affected by blood and plasma).

To simplify the analysis, we used a normalization of the calculated nanomolar data prior to PK analysis that compensates for fluorophore performance (e.g., microenvironment effects on fluorescence output) as well as for potential dosing and mouse size variability. Generally, the earliest possible acquisition time on the FMT 4000 imaging system is $1-5$ minutes (defined as the time of scan initiation plus the midpoint time for the scan; i.e., 2-6 minutes), but normalization requires an "extrapolated" $\mathrm{C}_{0}\left(\mathrm{C}_{0 \mathrm{E}}\right)$ In general, the $\mathrm{C}_{0 \mathrm{E}}$ will be higher than $\mathrm{C}_{1}$ and may differ somewhat from $1330 \mathrm{nM}$, depending on the overall fluorophore performance and clearance profile. The $\mathrm{C}_{0 \mathrm{E}}$ can be determined by a variety of curve-fitting methods (or even manual linear extrapolation from the first two acquired data points), but we used the PK Solver 2.0 Excel add-in (PK Solver) to determine $\mathrm{C}_{0 \mathrm{E}}$. Normalization of the measured data curves based on mathematical correction of the $\mathrm{C}_{0 \mathrm{E}}$ to the calculated $\mathrm{C}_{0}$ provided improvements in data robustness when again analyzed by PK Solver. Briefly, for normalization, all data points for an individual animal are multiplied by 1330 and divided by $\mathrm{C}_{0 \mathrm{E}}$, yielding that mouse's $\mathrm{PK}$ curve that now starts at a $\mathrm{C}_{0}$ value of $1330 \mathrm{nM}$ and has the appropriate changes over time. This process is repeated for all of the other individual mice. Normalized curves (individual or means of all animals) can be analyzed using any PK software. We used PK Solver for our analyses, choosing noncompartmental or compartmental analyses based on best fit of the data points. For consistency, identical normalization procedures were performed on blood fluorescence microplate data.

GFR Analysis. PK analysis for GFR680, which is very rapidly cleared ( $<1$ minute blood $t_{1 / 2}$ ), required a more challenging imaging procedure and quantitative approach, which was possible because GFR680 shows little in vivo alteration of fluorescence emission. Analysis relied on the accuracy of FMT quantification values rather than normalization, which means that very precise calibration of the system was essential. Scan fields were established that kept acquisition time to 2 minutes (i.e., use of $\sim 30$ scan points encompassing the thoracic region). The first 2-minute image acquisition was initiated immediately after probe injection. The optimal time points for scan initiation were $1,5,14,29$, and 44 minutes, with actual time points defined as the initiation time plus the midpoint of each 2-minute scan (i.e., 2, 6, 15, 30, and 45 minutes). For analysis, slightly oversized ROIs were placed around the heart to ensure the capture of all heart fluorescence. As described above, total heart picomolar values were again used (rather than nanomolar values) to avoid partial volume effects. All heart picomolar values were divided by $0.1 \mathrm{ml}$ to yield concentration in nanomoles. For PK/clearance analysis, a calculated $t_{0}$ was set as $1330 \mathrm{nM}$; for a normal mouse, a 50\%-60\% decrease was expected at $t=1$ minute relative to $t_{0}$. Since the clearance of inulin is so rapid, it was important to include the calculated time zero concentration $(1330 \mathrm{nM})$ to achieve good and consistent calculation of clearance results. A two-compartment curve fit and analysis generally worked very well and provided clearance values consistent with reports in the literature for normal female mice of this age and/or size, ranging from 200 to $300 \mu \mathrm{l} / \mathrm{min}$. Other mouse strains may or may not differ, but they will be internally consistent based on strain, sex, and/or size. Although GFR680 fluorescence appears to be unaffected by blood and provides objectively accurate quantification in a properly calibrated FMT system, recalibration or manual data adjustment of the FMT can be performed to ensure that appropriate GFR values are achieved in normal control mice.

PK Assessment by Microplate Assay. SKH1-E or BALB/c female mice were injected intravenously with $2 \mathrm{nmol}$ of each 
fluorescent agent. Terminal blood samples were collected by cardiac puncture (using heparinized syringes) from two to three mice at each time point after carbon dioxide asphyxiation. Blood was diluted 1:2 in DMSO to ensure capture of maximal fluorescent signal, and fluorescence was measured using a fluorescence plate reader (Molecular Devices, Sunnyvale, CA). The data were normalized to a standard curve prepared in blood/DMSO with known concentrations of the agent to quantify blood levels in nanomolar concentration. Results were normalized to the first collected time point, which was set as 1330 $\mathrm{nM}$ as with the in vivo imaging analysis. PK analysis was performed as described above.

Ex Vivo Plasma Creatine and BUN Assays. Plasma assays for creatinine (Creatinine Assay Kit MAK080; Sigma-Aldrich, St. Louis, MO) and BUN (QuantiChrom Urea Assay Kit DIUR-100; BioAssay Systems, Hayward, CA) were performed according to the manufacturers' instructions. Briefly, plasma samples were collected from sham, uninephrectomized, vehicle-treated, and CsA-treated mice and filtered through a cutoff filter with a molecular mass of $10 \mathrm{kDa}$. Samples $(5 \mu \mathrm{l})$ were assayed in duplicate in 96-well plates. Quantification was made in comparison with creatinine and BUN standard curves.

\section{Results}

A Fluorescence Tomographic Imaging Method for Measuring Heart Blood PK. The use of fluorescence tomography to acquire fluorescent heart images and the quantification of NIR fluorescent-labeled agents requires careful mouse preparation, proper study design, consistent animal positioning, and proper analysis to generate robust blood PK data. The first important consideration is to work with hairless or depilated mice (completed hair removal of front, sides, and back of thoracic region) to achieve proper transillumination required for tomographic imaging and reconstruction. A second consideration is that a unique laserbased fluorescence tomographic imaging system (e.g., the FMT 4000) is required to get proper 3D imaging of the heart. Third, mice should be injected with no more than $2 \mathrm{nmol}$ of the agent (as assessed by fluorescence) to limit blood levels to no more than $1.5 \mu \mathrm{M}$ concentrations. This injection dose avoids potential problems with fluorophore autoquenching at concentrations in blood $>2 \mu \mathrm{M}$ (i.e., a condition yielding falsely low readings), which would lead to abnormal PK curves and inaccurate quantification.

Figure 1 illustrates the concept of heart tomography PK and proper study design, which requires an initial acquisition time within 5 minutes of injection as well as additional acquisition time points that cover the full range of expected blood clearance kinetics. At $t_{0}, 100 \%$ of the injected conjugate is in the blood, including the heart, allowing a reference time point of objectively known blood concentration. Because the heart of a 25 -g mouse is known to contain $7 \%$ of the circulating blood, this makes it a useful location to noninvasively assess blood fluorescence through imaging. The first acquired time point $t_{1}$ (recommended as a 1- to 5-minute acquisition time) will show some small decline in signal, the magnitude of which depends on the clearance kinetics of the specific conjugate, and this reflects the loss of blood signal due to clearance and/or extravasation into tissue. This signal will decrease further over subsequent times, until the heart signal is below detection at the last time $\left(t_{\mathrm{n}}\right)$. To test the application of heart tomography for PK assessment, normal SKH1-E mice received intravenous injections of FRS680 (a folate-based probe that clears quickly through the kidneys), and mice were imaged longitudinally. As shown in Fig. 2A, a tomographic scan field was established in the thoracic region, and scanning/reconstruction for $t=2$ minute (Fig. 2B) revealed an upper torso pattern of the heart, carotids/jugular veins, and upper liver vasculature. An ellipsoidal ROI placed to capture the $3 \mathrm{D}$ heart showed accurate localization of the heart in the appropriate position in the mice. The complete array of longitudinal tomographic images from 1 minute to 24 hours (Fig. 2C) illustrates the kinetic changes in heart blood levels.

Quantitative assessment of the FRS680 imaging data set, performed as described in the Materials and Methods, was compared with blood fluorescence measured using microplate assays of sequential bleeds of a much larger cohort $(n=30)$ of FRS680-injected mice. Graphing of the PK data (Fig. 3, A and B) confirmed very similar curves for noninvasive imaging and blood assays $\left(r^{2}=0.96\right)$, and comparison of various PK parameters (Fig. 3C) using a three-compartment fit confirmed very similar results in blood half-lives, clearance, volume of distribution, and AUCs. The $t_{1 / 2 \alpha}$ measurement (2 minutes) was consistent with the major route of clearance through the kidneys, and the $t_{1 / 2 \beta}$ (26 minutes vs. 23 minutes for heart vs. blood, respectively) and $t_{1 / 2} \gamma$ (240 minutes vs. 102 minutes) measurements were consistent with some target-specific tissue retention.

Assessment of the Blood PK Profiles for Four Additional NIR Fluorescent-Labeled Molecules of Various Sizes. Four additional fluorescent conjugates, ranging in molecular mass from 1.3 to $150 \mathrm{kDa}$, were also tested by heart imaging to establish the performance range of FMT heart PK assessment (Fig. 4). PK analysis was performed

Non-invasive Pharmacokinetics Assessment by Heart Fluorescence Tomography

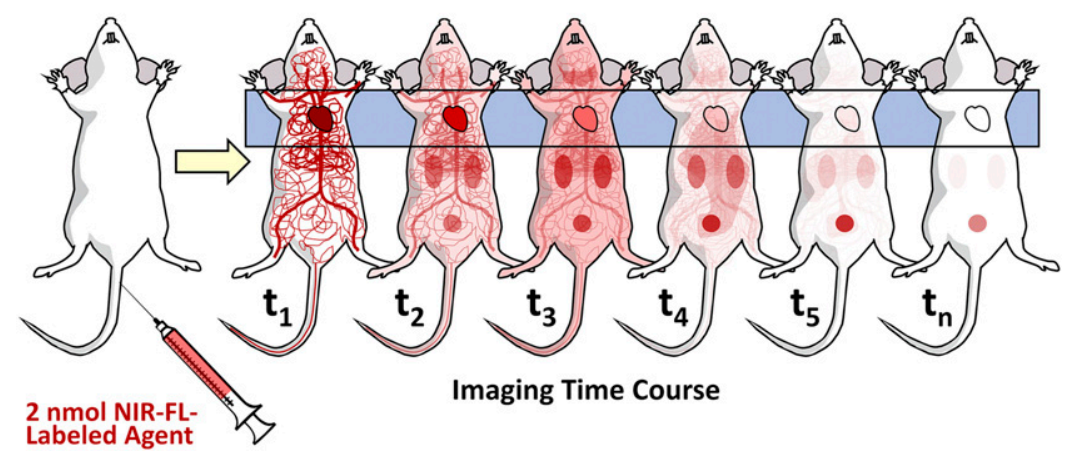

Fig. 1. Diagrammatic representation of the FMT heart tomography PK model. Mice are injected with NIR fluorescent-labeled agents at time 0 and imaged by FMT immediately, at the time $\left(t_{1}\right)$ when most of the fluorescence is in the circulation (as revealed by heart imaging). At defined times thereafter (dependent on the agent being studied), mice are imaged to assess the kinetic changes in heart signal that reflect overall blood PK. 


\section{A. Scan Field}

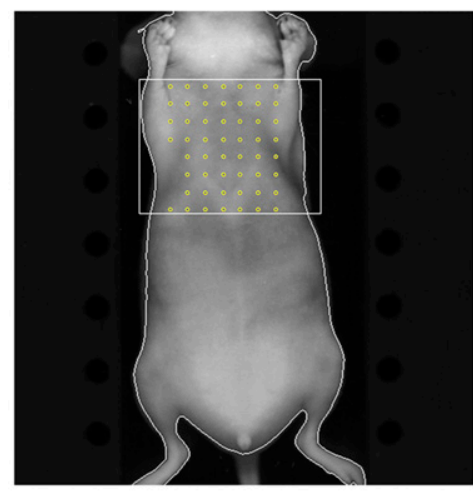

\section{B. Heart ROI Placement}

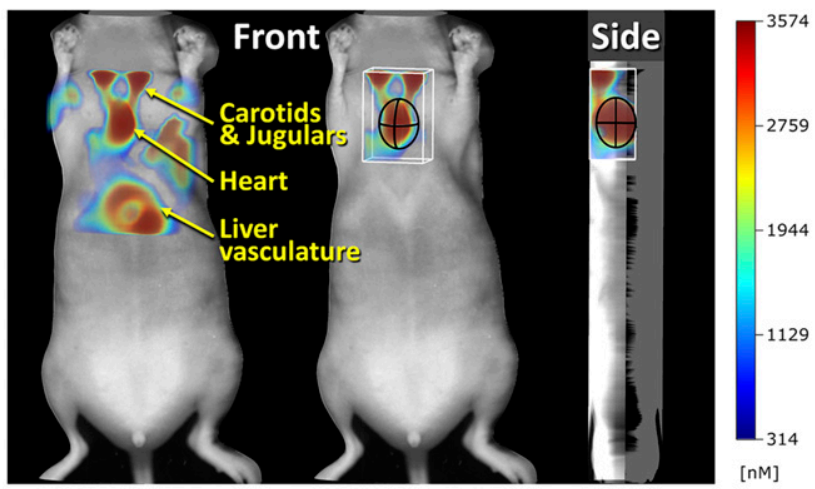

\section{Heart Imaging Time Course}

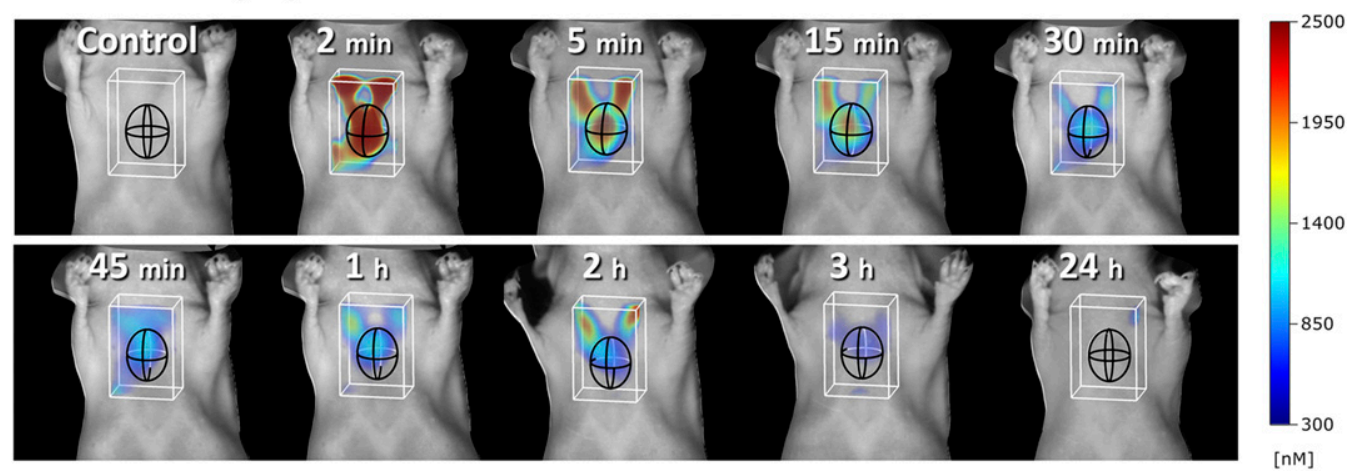

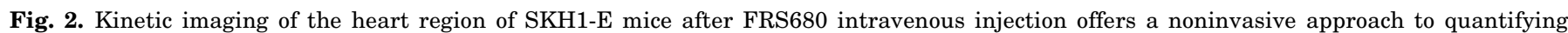

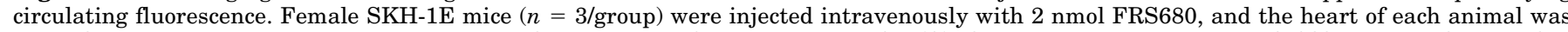

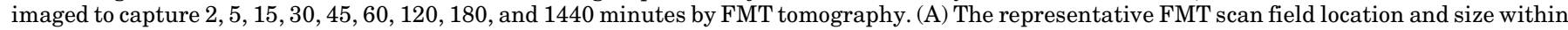

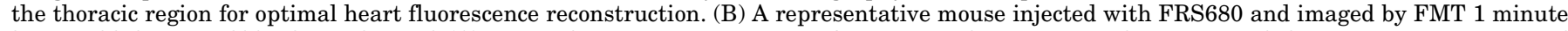

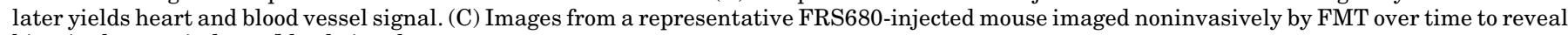
kinetic changes in heart/blood signal.

using noncompartmental, one-compartment, and twocompartment curve fitting to identify the best approach (i.e., the closest fit for the data) for each fluorescent conjugate. Two versions of the vascular probe AngioSense (AS680EX and AS750EX), both $70 \mathrm{kDa}$ and differing only modestly in structure, were measured longitudinally by heart tomography and by serial bleeds. Surprisingly, the two probes differed dramatically (by $\sim 7$ - to 10 -fold) in clearance rates and blood half-lives, with AS750 showing longer retention in vivo $\left(t_{1 / 2}=\right.$ 38 hours vs. 4 hours for AS680). In comparing the two methods for PK assessment, the curves were quite similar despite using only three mice per probe for heart imaging (with all time points acquired in each mouse), whereas 12 or 15 mice were used for blood assays (terminal bleeds of three mice for each time point). For both probes, there were excellent correlations between heart imaging and direct blood assay when comparing the PK curves $\left(r^{2}>0.90\right)$ as well as when comparing analytical readouts like half-life, AUC, clearance, and volume of distribution at steady state $\left(r^{2}=0.99\right)$. The $1.3-\mathrm{kDa}$ probe IS750 showed some minor differences when comparing heart imaging and direct blood assessment, with PK parameters differing by roughly 2 -fold, although PK curves correlated well and differed in half-life only by 30 minutes. Application of the two PK assessment methods to NIR fluorescent-labeled control mouse IgG1 ( $150 \mathrm{kDa})$ yielded very similar results with high correlations of curves and various PK measures.
Measuring GFR Using GFR680 Heart PK Imaging. Clinicians mostly rely on indirect calculation of GFR based on plasma creatinine; however, direct GFR measurement (quantifying clearance of injected inulin) is the gold standard for detecting kidney changes associated with drug-induced injury or disease. The rate of clearance of labeled inulin from the blood is equivalent to the filtration rate at the kidneys' glomeruli, as there is no subsequent tubular reabsorption. GFR measurement relies on two established approaches: 1) continuous inulin intravenous infusion with timed urine collection, or 2) a single bolus intravenous injection with timed blood collection ( $\mathrm{Qi}$ and Breyer, 2009). The first approach is impractical in mice because steady-state plasma levels are difficult to achieve, and the approach can overestimate GFR due to the potential for nonrenal clearance of inulin that arises with continuous infusion (Orlando et al., 1998). The second approach is simple and practical with little or no contribution of nonrenal clearance, but it requires collection of several timed blood samples, which can require multiple cohorts of mice for preclinical application.

We have developed an NIR fluorescent-labeled form of inulin (GFR680) for in vivo use in mouse intravenous bolus GFR measurement. Figure 5 shows an experiment used to characterize GFR680 performance, focusing on heart imaging for the purposes of PK measurement but including kidney tomography and two-dimensional epifluorescence imaging of 


\section{A PK Measurement Approaches}

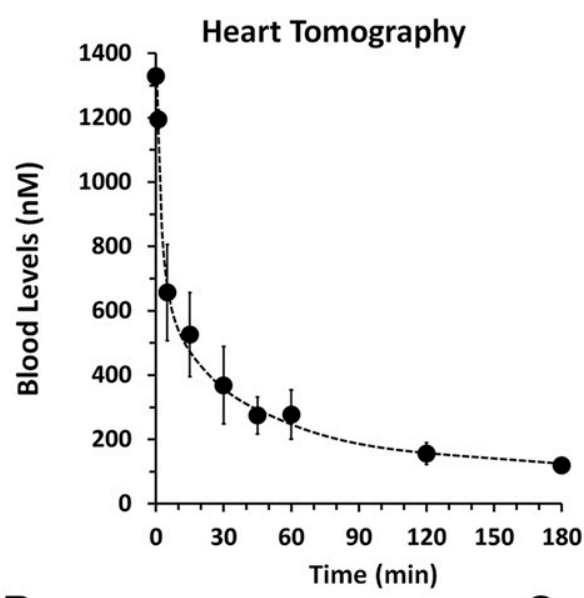

B

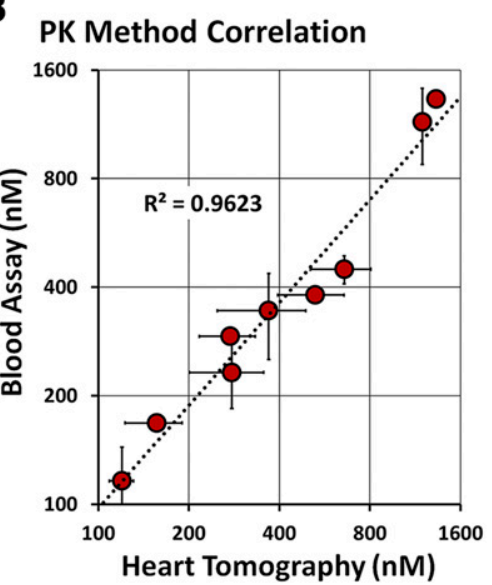

C

PK Parameters (Heart Imaging vs Blood Assay)

\begin{tabular}{|c|c|c|c|}
\hline Parameter & Unit & Heart & Blood \\
\hline $\mathbf{t}_{1 / 2}$ Alpha & $\min$ & 2 & 2 \\
\hline$t_{1 / 2}$ Beta & $\min$ & 26 & 23 \\
\hline $\mathrm{t}_{1 / 2}$ Gamma & $\min$ & 240 & 102 \\
\hline co & $\mathrm{nM}$ & 1362 & 1376 \\
\hline v & $\mathrm{ml}$ & 1.5 & 1.5 \\
\hline $\mathrm{CL}$ & $\mathrm{ml} / \mathrm{min}$ & 0.023 & 0.034 \\
\hline V2 & $\mathrm{ml}$ & 1.3 & 3.3 \\
\hline CL2 & $\mathrm{ml} / \mathrm{min}$ & 0.224 & 0.36 \\
\hline V3 & $\mathrm{ml}$ & 3.6 & 0.0 \\
\hline CL3 & $\mathrm{ml} / \mathrm{min}$ & 0.035 & 0 \\
\hline AUC $0-t$ & $\mathrm{nM} M^{*} \min$ & $4.6 \mathrm{E}+04$ & $4.3 E+04$ \\
\hline AUC 0 -inf & $\mathrm{nM}^{*} \min$ & $8.7 E+04$ & $6.0 E+04$ \\
\hline AUMC & $n M^{\star} \min ^{\wedge} 2$ & $2.41 \mathrm{E}+07$ & $8.42 E+06$ \\
\hline MRT & $\min$ & 279 & 141 \\
\hline Vss & $\mathrm{ml}$ & 6.5 & 4.7 \\
\hline
\end{tabular}

Fig. 3. FRS680 heart PK analysis and comparison with $\mathrm{PK}$ assessment by serial bleeds and microplate assay. (A) Quantitative PK curve established noninvasively by FMT heart imaging of SKH1-E mice $(n=3)$ was compared with a parallel study in which multiple cohorts of mice $(n=30)$ were serially bled and samples were assayed ex vivo in comparison with a standard curve. (B) Correlation between heart tomography and blood assay results. (C) Various PK parameters were assessed using PK Solver, with a good correlation $(\mathrm{r} 2=0.99)$ between quantitative PK parameters AUMC, area under the first moment curve; CL, clearance; MRT, mean residence time; $\mathrm{V}$, volume of distribution. bladder signal to better understand clearance behavior. FMT scans of the heart (Fig. 5A, left image) were used to detect and quantify blood levels of GFR680 at multiple time points after a bolus intravenous injection of $2 \mathrm{nmol}$ probe. After the injection in five normal SKH-1E mice, FMT images were acquired at $2,6,15,30$, and 45 minutes postinjection (Fig. 5B, upper panel). Clearance rates for the five mice in this study were calculated using a two-compartment curve fitting, yielding an average rate of $240 \pm 50 \mu \mathrm{l} / \mathrm{min}$ in this study in normal mice (Fig. 5C). We examined the possibility of using either kidney tomography (Fig. 5A, right image) or bladder epifluorescence imaging (Fig. 5A, left image) as potential methods to more easily assess GFR680 clearance as single time-point acquisitions. Although both kidney and bladder signal generally increased over time in most mice (Fig. 5B, middle and lower panels), the absolute amounts of fluorescence were highly variable between animals and were impacted substantially by bladder voiding. Figure 5D shows that two of five mice lost most of their kidney fluorescence due to bladder voiding early in the imaging process. These two mice also were delayed or lower in bladder signal (Fig. 5E). Figure $5 \mathrm{~F}$ represents a tabular summary of five separate studies measuring GFR using heart tomography, showing an average GFR value for normal mice at $240 \mu \mathrm{l} / \mathrm{min}$ with $\sim 8 \%$ interassay variability. This average value falls within the typical range of published mouse inulin-based assessment of GFR values in anesthetized mice $(229-325 \mu \mathrm{l} / \mathrm{min})$ as summarized by Qi et al. (2004). A subset of our heart tomography data was also compared with timed ex vivo blood assays (assessed by microplate assays) of mice receiving either GFR680 or inulin-fluorescein isothiocyanate (Supplemental Fig. 1), with excellent correlations of heart tomography to microplate assays (Fig. 5F).

Validating the Heart Tomography GFR Method in UNX Mice. After establishing normal ranges of GFR determined by heart tomography, it was important to validate this assay by altering GFR in a defined manner. Surgical removal of a single kidney has an expected dramatic effect on overall kidney filtration that has been studied in both experimental animals and humans. The remaining kidney undergoes compensatory changes, including hypertrophy and/or hyperplasia of glomeruli and tubules, increased metabolic activity, and an increase in urinary output, over time resulting in improved glomerular filtration (Fine, 1986). In UNX rats, the expected 50\% decline in GFR is followed by some functional compensation after 2 days, leading to only a 25\% deficiency in GFR over the long term (Chamberlain and Shirley, 2007). In mice, however, GFR declines by $50 \%$ immediately after a unilateral nephrectomy but recovers very little over 52 weeks to $\sim 41 \%$ deficiency (Jani et al., 2006). This suggests that functional kidney measurements within 1 week in UNX mice would reflect near maximal loss of GFR function. 
AngioSense 680EX

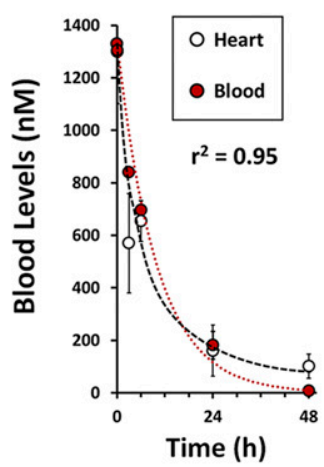

AngioSense 750EX

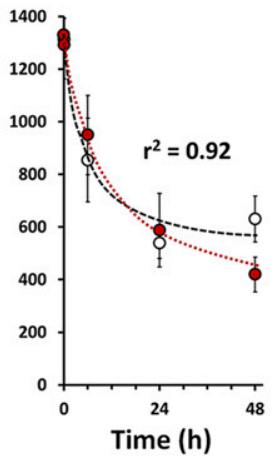

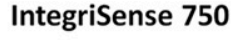

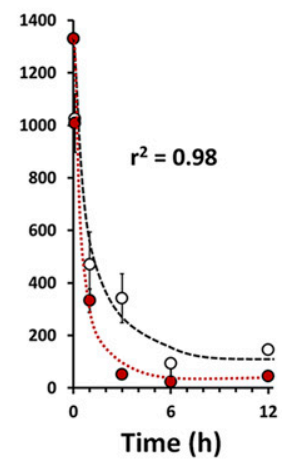

IgG1-VivoTag680XL

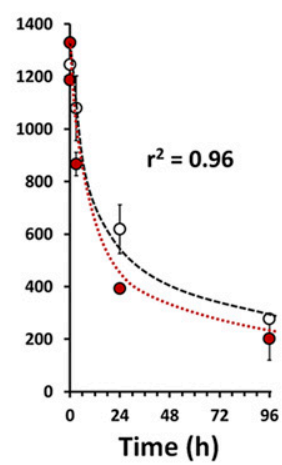

\begin{tabular}{|c|c|c|c|c|c|c|c|c|}
\hline $\begin{array}{r}\text { Specificity } \\
\text { Molecular Weight } \\
\text { PK analysis Model }\end{array}$ & \multicolumn{2}{|c|}{$\begin{array}{c}\text { Vascular Probe } \\
70 \mathrm{kDa} \\
1 \text { Compartment }\end{array}$} & \multicolumn{2}{|c|}{$\begin{array}{c}\text { Vascular Probe } \\
70 \text { kDa } \\
\text { Noncompartmental }\end{array}$} & \multicolumn{2}{|c|}{$\begin{array}{c}\alpha \mathrm{V} \beta 3 \text { Integrin Probe } \\
1.28 \mathrm{kDa} \\
1 \text { Compartment }\end{array}$} & \multicolumn{2}{|c|}{$\begin{array}{c}\text { Immunoglobulin } \\
150 \mathrm{kDa} \\
\text { Noncompartmental }\end{array}$} \\
\hline & Heart & Blood & Heart & Blood & Heart & Blood & Heart & Blood \\
\hline$t_{1 / 2}(h)$ & 4.4 & 6.3 & 37.8 & 43.9 & 1.0 & 0.5 & 46.7 & 38.5 \\
\hline AUC 0-t (pmol/ml*h) & 7824 & 11305 & 41785 & 43670 & 1764 & 893 & 53608 & 37691 \\
\hline $\mathrm{CL}(\mu \mathrm{l} / \mathrm{min})$ & 4.2 & 2.8 & 0.5 & 0.4 & 18.7 & 37.3 & 0.5 & 0.7 \\
\hline Vss (ml) & 1.6 & 1.5 & 1.5 & 1.5 & 1.7 & 1.6 & 1.6 & 1.7 \\
\hline
\end{tabular}

Fig. 4. Heart PK analysis of three NIR fluorescent imaging probes and NIR fluorescent-labeled IgG1. The upper panel shows quantitative PK curves for three probes established noninvasively by FMT heart imaging of SKH1-E mice $(n=3)$ compared with parallel studies in which multiple cohorts of mice $(n=30)$ were serially bled and samples were quantified ex vivo based on a standard curve. The same comparison was made for IgG1- VT680XL in depilated female BALB/c mice. The lower panel is a tabular summary of various PK parameters that were assessed using PK Solver for both heart imaging and blood assay data. CL, clearance; Vss, volume of distribution at steady state.

To assess our heart imaging methodology, UNX SKH1-E female mice were imaged ( 7 days after uninephrectomy) at 2 , $6,15,30$, and 45 minutes using GFR680, and the calculated clearance rates were compared with normal (sham surgery) mice. Figure $6 \mathrm{~A}$ shows heart region tomographic images over time, illustrating obvious differences at most time points. Graphical representation of TrueQuant quantification for both individual mice (Fig. 6B) and group averages (Fig. 6C) reveals a clear impairment or slowing of clearance kinetics with statistical differences observed at 15, 30, and 45 minutes. Clearance rates derived from these curves determined a $52 \%$ decrease in UNX group GFR relative to that of sham controls (Fig. 6D), with accompanying more modest changes $(\sim 1.5 \times$ increases $)$ in plasma creatinine and BUN (Fig. 6E).

Measuring CsA-Induced Impairment of GFR. CsA is well characterized for its ability to cause kidney injury, inducing acute and chronic renal dysfunction, hemolytic-uremic syndrome, hypertension, tubular acidosis, juxtaglomerular apparatus hypertrophy and hyperplasia, increased kidney renin, and progressive renal interstitial fibrosis (Burdmann et al., 2003). We used CsA treatment of mice based on the protocol of Puigmule et al. (2009) as another means to validate FMT heart tomography for assessing GFR changes. Briefly, female SKH-1E mice were injected subcutaneously with $\mathrm{CsA}(80 \mathrm{mg} / \mathrm{kg}$ per day) or vehicle (olive oil) once per day for 14 days. Mice were then injected intravenously with $2 \mathrm{nmol}$ GFR680, and the heart of each animal was imaged at 2, 6, 15, 30, and 45 minutes by fluorescent tomography. Graphical representation of TrueQuant quantification (Fig. 7A) shows impairment of GFR as a clear curve shift in blood fluorescence kinetics. Clearance rates derived from these curves determined a 47\% decrease in the CsA group
GFR relative to that of vehicle controls (Fig. 7B), and there were also significant changes $(\sim 2.5 \times$ increases $)$ in plasma creatinine and BUN (Fig. 7C).

\section{Discussion}

Fluorescence optical imaging remains a young imaging modality but has capabilities that can support a wide range of preclinical therapeutic areas of research (Kossodo et al., 2010; Peterson et al., 2010; Ackermann et al., 2011; Zhang et al., 2012; Felix et al., 2015; Peterson, 2016; Vasquez and Peterson, 2017). Advances in photonic technology and reconstruction algorithms (Meyer et al., 2007; Lasser et al., 2008; Mohajerani et al., 2009; Mohajerani and Ntziachristos, 2016) have even taken optical imaging beyond standard twodimensional epifluorescence imaging into the realm of $3 \mathrm{D}$ tomographic imaging for localization and quantification of fluorescence in deep tissue. This has opened the door to more challenging imaging applications, including brain, heart, liver, and lung imaging (Korideck and Peterson, 2009; Clapper et al., 2011; Lin et al., 2012; Eaton et al., 2013). Coregistered tomographic FMT and positron emission tomography (PET) data sets agreed very well spatially and quantitatively (Nahrendorf et al., 2010), supporting the accuracy of FMT imaging as a nonradioactive surrogate for preclinical PET imaging applications. Indeed, one of the most important PET imaging applications, whole-body biodistribution, is a method that was established in 2011 as a robust FMT application (Vasquez et al., 2011), with large and small fluorescent agents showing differential kinetics of fluorescence distribution to, and clearance from, major tissues and organs. Subsequent mouse studies determined that tomographic heart 
A FMT Imaging Strategy

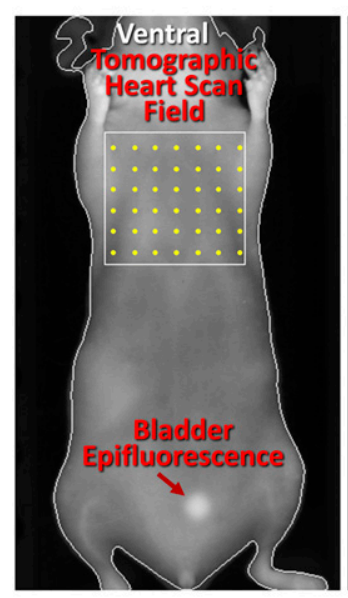

C Heart (blood)

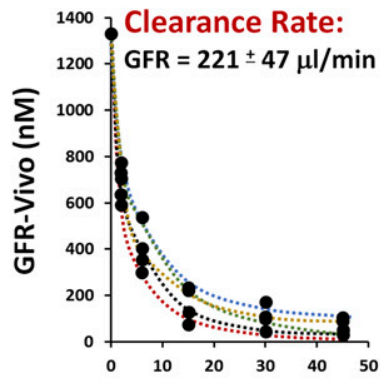

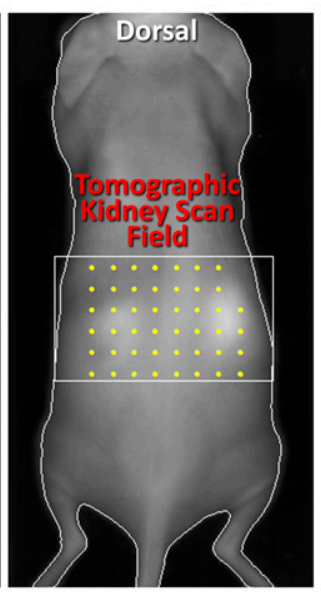

D Kidney

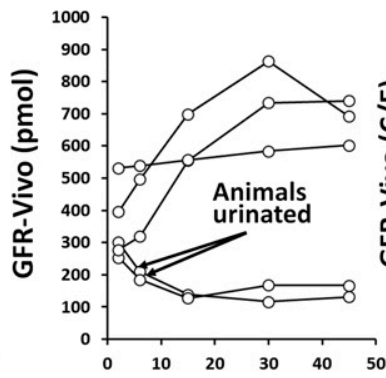

Time Post-Injection ( $\mathrm{min})$

B Heart, Kidney, Bladder Images

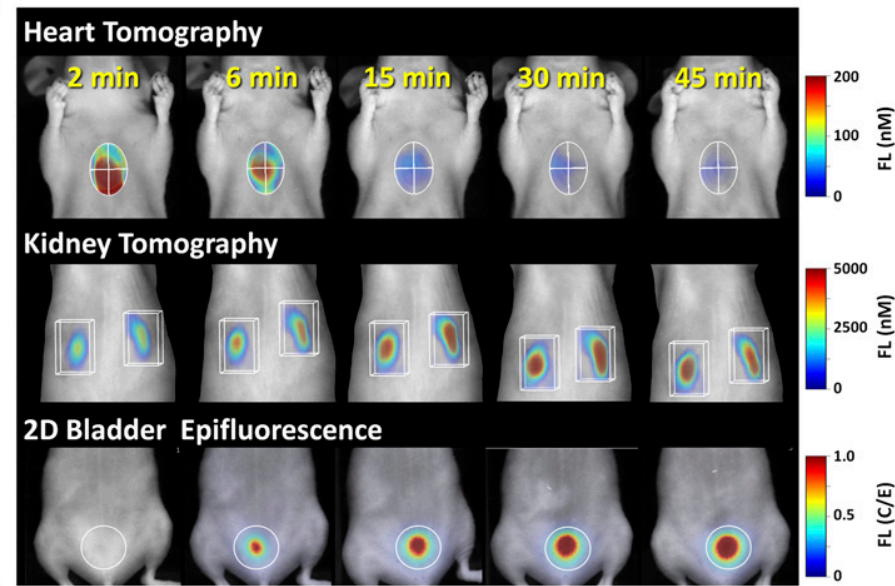

E Bladder

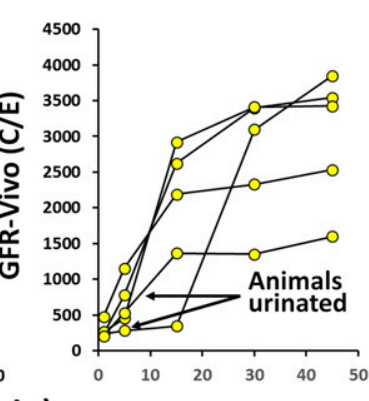

F Heart GFR Summary

\begin{tabular}{|c|c|}
\hline Parameter & Comment \\
\hline Study \# & 5 studies \\
\hline Curve Fit & $\begin{array}{c}\text { Bolus IV } \\
2 \mathrm{cmpt} \text { fit }\end{array}$ \\
\hline Ave. GFR ( $\mu \mathrm{l} / \mathrm{min}$ ) & $240 \pm 21$ \\
\hline Inter-assay CV & $8 \%$ \\
\hline $\begin{array}{c}\text { Corr. to microplate } \\
\text { assay (GFR-Vivo) }\end{array}$ & $\mathrm{r}^{2}=0.93$ \\
\hline $\begin{array}{c}\text { Corr. to microplate } \\
\text { assay (Inulin-FITC) }\end{array}$ & $\mathrm{r}^{2}=0.97$ \\
\hline
\end{tabular}

Fig. 5. GFR assessment in normal mice by FMT heart imaging. Normal female SKH-1E mice ( $n=5$ /group) were injected intravenously with 2 nmol GFR680, and the heart of each animal was imaged over time by FMT 4000 tomography. (A) Ventral and dorsal imaging strategies illustrating position and size of tomographic scan field for the heart (left) and kidneys (right). Parallel acquired ventral epifluorescence was used to measure bladder signal (left). (B) Representative images of heart tomography (upper), kidney tomography (middle), and bladder epifluorescence (lower), showing the kinetic change in fluorescence in these regions. (C) Quantitative PK graph of data established noninvasively by FMT heart imaging of SKH1-E mice, showing each individual mouse's blood PK curve. GFR was determined using two-compartment fits for each mouse using PK Solver. (D) Quantitative kidney fluorescence kinetics established noninvasively by FMT imaging. (E) Quantitative bladder epifluorescence kinetics established noninvasively by two-dimensional imaging in units of fluorescent counts per energy. (F) Tabular summary of results from five studies in normal mice, showing average group results and interassay CV. C/E, count per energy; CV, coefficient of variation; FITC, fluorescein isothiocyanate; FL, fluorescence; IV, intravenous.

fluorescence results generally correlated quite well with blood PK assessment; however, epifluorescence approaches in mice (heart, superficial veins, eyes) were poor or almost completely ineffective (Peterson et al., unpublished observations). This means that fluorescence heart tomography is a useful optical approach to the noninvasive assessment of blood PK, offering some clear benefits in reduction of animals and/or improved ease of use compared with other imaging and nonimaging approaches.

The data in this report examine fluorescent conjugates with a molecular mass from 1.3 to $150 \mathrm{kDa}$, with comparable PK results seen in parallel heart tomography and serial bleed assays (Figs. 2-4). The imaging approach offers two advantages over microplate assays: 1) full kinetic curves for each individual animal and 2) the ability to use only three to five mice. There are also some advantages of this imaging approach over blood microsampling approaches, which can also decrease animal use, in that there is no sample collection, manipulation, or additional analytical acquisition required. Data are generated from the heart imaging data sets using simple analysis tools. The major application for FMT technology in PK imaging is likely to be assessing antibodies or large protein therapeutics, since the NIR fluorophore reporters used tend to be large ( $1000 \mathrm{Da}$ ) but would have minimal effect on the size of high molecular weight proteins like antibodies. Indeed, a preliminary report showed that heart tomography with NIR fluorescent-labeled therapeutic antibodies can generate useful PK readouts (Giddabasappa et al., 2016). In contrast, NIR fluorophore conjugation significantly increases the size of small molecule drugs, which would then likely result in altered PK and biodistribution (i.e., generating data not easily extrapolated to the unconjugated parent molecule). However, even for large proteins, researchers must take care to not overlabel with fluorophores, which can risk changing the PK of the parent molecule. In general, a 1-to-1 labeling ratio works well, but we are also currently exploring lower molecular weight NIR fluorophores to further minimize potential PK-altering conjugation effects.

A second important use of FMT heart imaging, applicable to imaging probes of any size, is fluorescent probe development and characterization, in which the conjugate itself is the relevant agent being studied. Understanding PK is essential in probe design as well as in determining the earliest possible 


\section{A FMT Heart Fluorescence Images}

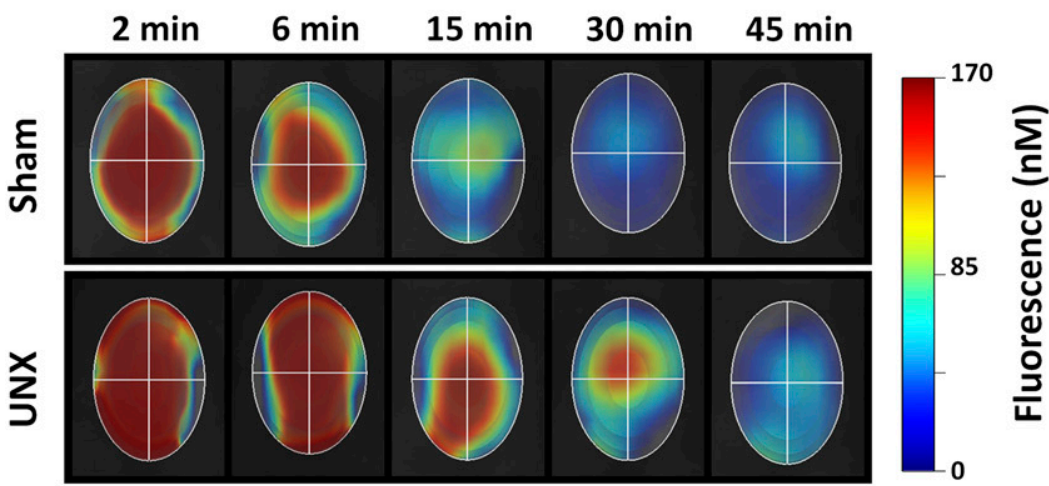

B

B Individual Heart Data

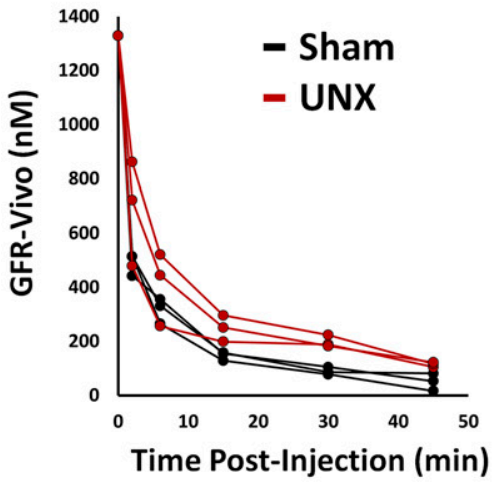

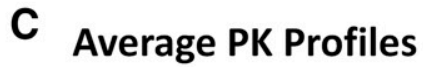

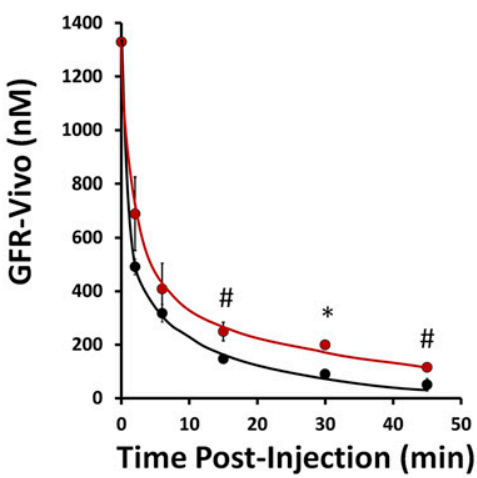

Fig. 6. Unilateral nephrectomy-induced effects on kidney GFR function. Seven days after uninephrectomy, female SKH-1E mice ( $n=3$ /group) were injected intravenously with 2 nmol GFR680 and the heart of each animal was imaged over time by FMT 4000 tomography. (A) Heart tomography images from representative sham and UNX mice, illustrating obvious differences in the clearance kinetics. (B) Line graphs of heart fluorescence kinetics for each individual sham and UNX mouse. (C) Line graphs for group averages of sham and UNX mice with curve fitting. (D) Clearance rates for each group based on FMT heart PK were calculated using PK Solver. (E) Plasma creatinine and BUN were assessed, revealing modest UNX-induced increases. Asterisks and hatchmarks indicate statistically significant differences between sham and UNX groups $\left(* P<0.01 ;{ }^{*} P<0.05\right)$. Dotted lines highlight the basal background levels for creatinine and BUN based on the sham control group.

\section{GFR (clearance rate)}

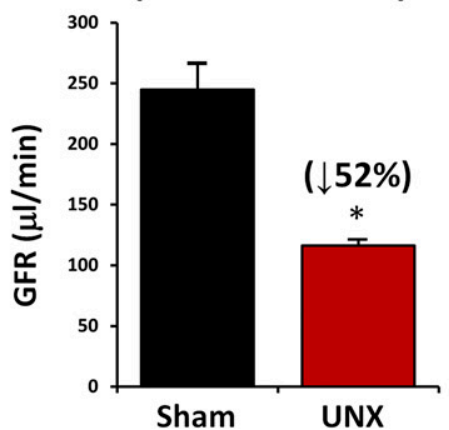

\section{E Creatinine and BUN}

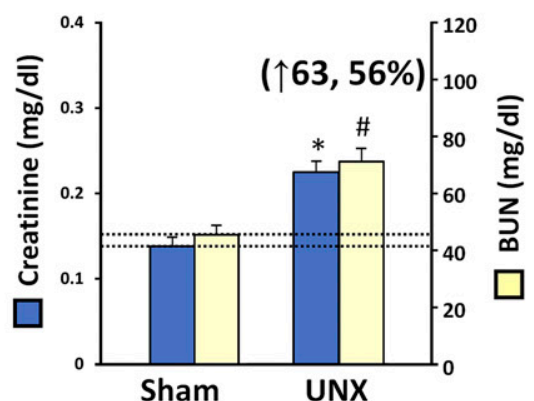

imaging windows. Some imaging applications for fluorescent probes may also benefit from PK assessment (e.g., correcting biodistribution imaging data to better understand vascular contribution versus tissue extravasation at sites of interest) (Vasquez et al., 2011). For vascular probes, kinetic imaging with blood-level correction can add another dimension to understanding vascularity, vascular damage, edema, and angiogenesis in a variety of inflammatory or disease states. Rates of tissue extravasation and clearance in some instances may more sensitively characterize physiologic changes in tissue (e.g., in treatments affecting edema or angiogenesis).

Finally, PK assessment of labeled inulin (Figs. 5-7) offers a very unique approach to assessing damage-induced alterations in GFR (Qi et al., 2004, 2005; Bivona et al., 2011; Rieg, 2013), and noninvasive heart tomography of an NIR fluorescent-labeled inulin, in particular, offers the advantage of requiring very few mice. The best use of this mouse GFR method is likely in early drug discovery, providing early risk assessment to help prioritize research on compounds with good safety profiles. Our heart imaging approach here has been validated using both a uninephrectomy model and CsA-induced kidney injury, with additional comparison with conventional plasma creatinine and BUN assays. The results for normal GFR are consistent with numbers reported in the literature data (Qi et al., 2004, 2005; Bivona et al., 2011; Rieg, 2013). It is interesting to note that we did not observe obvious GFR compensation in UNX mice at 7 days (which theoretically should have shown $<50 \%$ GFR inhibition if compensation was occurring); however, the timing of GFR compensation after uninephrectomy in mice has not been characterized. A similar GFR alteration ( $45 \%$ decrease) was seen with 2 weeks of CsA dosing, and some additional preliminary studies 
A Average PK Profiles

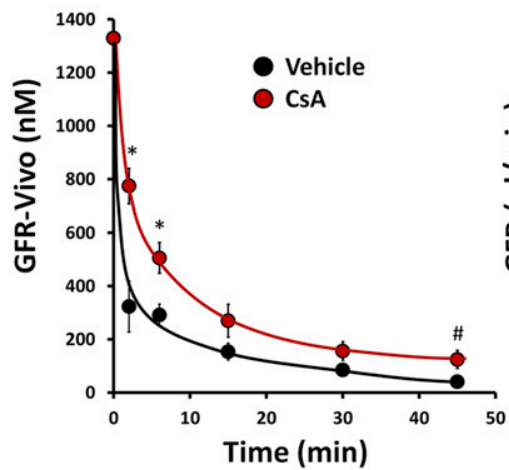

B GFR (clearance rate)

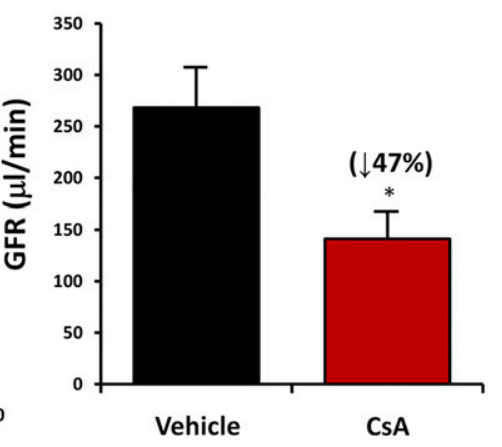

C Creatinine and BUN

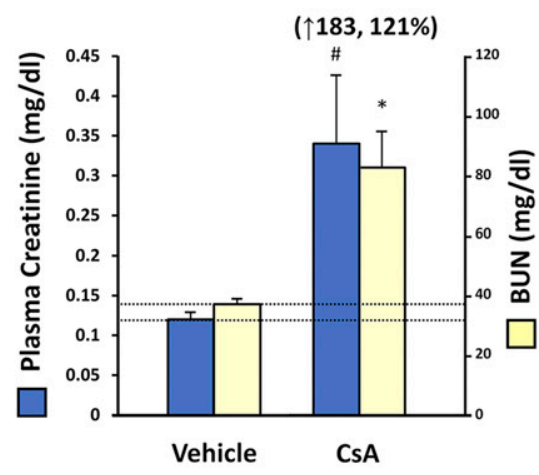

Fig. 7. CsA-induced effects on kidney GFR function. Female SKH-1E mice ( $n=5 /$ group) were treated with $100 \mu \mathrm{l} \mathrm{CsA}$ ( $80 \mathrm{mg} / \mathrm{kg}$ per day formulated in olive oil) or vehicle (olive oil) injected subcutaneously for 14 days. Mice were then injected intravenously with 2 nmol GFR680 and the heart of each animal was imaged at 1, 5, 15, 30, and 45 minutes by FMT 4000 tomography. (A) Average heart levels of GFR680 in vehicle- and CsA-treated mice. (B) Average calculated GFR for each group. (C) Plasma creatinine and BUN were assessed, revealing CsA-induced increases. Asterisks and hatchmarks indicate statistically significant differences between vehicle and CsA groups $\left({ }^{*} P<0.01 ;{ }^{\#} P<0.05\right)$. Dotted lines highlight the basal background levels for creatinine and BUN based on the vehicle group.

suggest a greater magnitude of GFR inhibition at early time points (Peterson, unpublished observations), suggesting that some GFR compensation may occur later despite ongoing treatment. Further studies are in progress to examine the effects of other drugs as well as the kinetics of kidney compensatory functional recovery after tissue injury. The heart imaging method is not only amenable to kinetic imaging to assess GFR, but it can also be used in a longitudinal fashion such as repeated injection of GFR680 (with kinetic imaging) over many days of drug dosing. Unfortunately, due to the challenges of fluorescent tomography, it is not possible to perform heart imaging studies in rats. This is a significant limitation; however, future advances in the technology with likely enable rat GFR assessment by fluorescent imaging, which could offer significant advantages over microcomputed tomography (which involves known kidney toxicity of contrast agents) and liquid chromatography-tandem mass spectrometry analysis of dried blood spots (labor-intensive analysis) for measuring kidney function.

In conclusion, we have demonstrated the ability of noninvasive FMT heart imaging to visualize and quantify blood $\mathrm{PK}$ in a robust and consistent manner with the need for very few animals. The consistency of the quantitative tomography, as well as its excellent correlation with ex vivo assessment of blood kinetics, provides a powerful tool for assessing large molecular weight biotherapeutics and various novel imaging probes. Application of this method to GFR680 provides a noninvasive approach to GFR measurements in models of kidney disease, dysfunction, and drug-induced injury.

\section{Authorship Contributions}

Participated in research design: Bao, Vasquez, Peterson.

Conducted experiments: Bao, Vasquez, Zhang.

Contributed new reagents or analytic tools: Ho, Delaney, Peterson. Performed data analysis: Bao, Peterson.

Wrote or contributed to the writing of the manuscript: Bao, Vasquez, Rajopadhye, Peterson.

\section{References}

Ackermann M, Carvajal IM, Morse BA, Moreta M, O’Neil S, Kossodo S, Peterson JD, Delventhal V, Marsh HN, Furfine ES, et al. (2011) Adnectin CT-322 inhibits tumor growth and affects microvascular architecture and function in Colo205 tumor xenografts. Int $J$ Oncol 38:71-80.

Balls M (2015) 16. The three Rs: the way forward. Altern Lab Anim 43:25-27.

Bivona BJ, Park S, and Harrison-Bernard LM (2011) Glomerular filtration rate determinations in conscious type II diabetic mice. Am J Physiol Renal Physiol 300: F618-F625.

Burdmann EA, Andoh TF, Yu L, and Bennett WM (2003) Cyclosporine nephrotoxicity. Semin Nephrol 23:465-476.

Chamberlain RM and Shirley DG (2007) Time course of the renal functional response to partial nephrectomy: measurements in conscious rats. Exp Physiol 92:251-262. Clapper ML, Hensley HH, Chang WC, Devarajan K, Nguyen MT, and Cooper HS (2011) Detection of colorectal adenomas using a bioactivatable probe specific for matrix metalloproteinase activity. Neoplasia 13:685-691.

Eaton VL, Vasquez KO, Goings GE, Hunter ZN, Peterson JD, and Miller SD (2013) Optical tomographic imaging of near infrared imaging agents quantifies disease severity and immunomodulation of experimental autoimmune encephalomyelitis in vivo. $J$ Neuroinflammation 10:138

Felix DD, Gore JC, Yankeelov TE, Peterson TE, Barnes S, Whisenant J, Weis J, Shoukouhi S, Virostko J, Nickels M, et al. (2015) Detection of breast cancer microcalcification using $(99 \mathrm{~m}) \mathrm{Tc}-\mathrm{MDP}$ SPECT or osteosense 750EX FMT imaging. Nucl Med Biol 42:269-273.

Fine L (1986) The biology of renal hypertrophy. Kidney Int 29:619-634.

Giddabasappa A, Gupta VR, Norberg R, Gupta P, Spilker ME, Wentland J, Rago B, Eswaraka J, Leal M, and Sapra P (2016) Biodistribution and targeting of anti-5T4 antibody-drug conjugate using fluorescence molecular tomography. Mol Cancer Ther 15:2530-2540.

Jani A, Wang W, Faubel S, Falk S, Ljubanovic D, Dursun B, and Edelstein CL (2006) Ischemic acute renal failure following nephrectomy impairs long-term renal function. Transplantation 81:800-803.

Korideck H and Peterson JD (2009) Noninvasive quantitative tomography of the therapeutic response to dexamethasone in ovalbumin-induced murine asthma J Pharmacol Exp Ther 329:882-889.

Kossodo S, Pickarski M, Lin SA, Gleason A, Gaspar R, Buono C, Ho G, Blusztajn A, Cuneo G, Zhang J, et al. (2010) Dual in vivo quantification of integrin-targeted and protease-activated agents in cancer using fluorescence molecular tomography (FMT). Mol Imaging Biol 12:488-499.

Lasser T, Soubret A, Ripoll J, and Ntziachristos V (2008) Surface reconstruction for free-space 360 degrees fluorescence molecular tomography and the effects of animal motion. IEEE Trans Med Imaging 27:188-194.

Lin SA, Patel M, Suresch D, Connolly B, Bao B, Groves K, Rajopadhye M, Peterson JD, Klimas M, Sur C, et al. (2012) Quantitative longitudinal imaging of vascular inflammation and treatment by ezetimibe in apoE mice by FMT using new optical imaging biomarkers of cathepsin activity and $\alpha(\mathrm{v}) \beta(3)$ integrin. Int $J$ Mol Imaging 2012:189254

Meyer H, Garofalakis A, Zacharakis G, Psycharakis S, Mamalaki C, Kioussis D, Economou EN, Ntziachristos V, and Ripoll J (2007) Noncontact optical imaging in mice with full angular coverage and automatic surface extraction. Appl Opt 46: 3617-3627.

Mohajerani P, Adibi A, Kempner J, and Yared W (2009) Compensation of optical heterogeneity-induced artifacts in fluorescence molecular tomography: theory and in vivo validation. J Biomed Opt 14:34021.

Mohajerani P and Ntziachristos V (2016) An inversion scheme for hybrid fluorescence molecular tomography using a fuzzy inference system. IEEE Trans Med Imaging 35:381-390.

Nahrendorf M, Keliher E, Marinelli B, Waterman P, Feruglio PF, Fexon L, Pivovarov M, Swirski FK, Pittet MJ, Vinegoni C, et al. (2010) Hybrid PET-optical imaging using targeted probes. Proc Natl Acad Sci USA 107:7910-7915.

Orlando R, Floreani M, Padrini R, and Palatini P (1998) Determination of inulin clearance by bolus intravenous injection in healthy subjects and ascitic patients: 
equivalence of systemic and renal clearances as glomerular filtration markers. $\mathrm{Br}$ $J$ Clin Pharmacol 46:605-609.

Peterson JD (2016) Noninvasive in vivo optical imaging models for safety and toxicity testing, in Nutraceuticals: Efficacy, Safety and Toxicity (Gupta RC ed) pp 305-317, Academic Press, London.

Peterson JD, Labranche TP, Vasquez KO, Kossodo S, Melton M, Rader R, Listello JT, Abrams MA, and Misko TP (2010) Optical tomographic imaging discriminates between disease-modifying anti-rheumatic drug (DMARD) and non-DMARD efficacy in collagen antibody-induced arthritis. Arthritis Res Ther 12:R105.

Puigmulé M, López-Hellin J, Suñé G, Tornavaca O, Camaño S, Tejedor A and Meseguer A (2009) Differential proteomic analysis of cyclosporine A-induced toxicity in renal proximal tubule cells. Nephrol Dial Transplant 24:2672-2686.

Qi Z and Breyer MD (2009) Measurement of glomerular filtration rate in conscious mice. Methods Mol Biol 466:61-72.

Qi Z, Fujita H, Jin J, Davis LS, Wang Y, Fogo AB, and Breyer MD (2005) Characterization of susceptibility of inbred mouse strains to diabetic nephropathy. $D i$ abetes 54:2628-2637.

Qi Z, Whitt I, Mehta A, Jin J, Zhao M, Harris RC, Fogo AB, and Breyer MD (2004) Serial determination of glomerular filtration rate in conscious mice using FITCinulin clearance. Am J Physiol Renal Physiol 286:F590-F596.

Rieg T (2013) A high-throughput method for measurement of glomerular filtration rate in conscious mice. $J$ Vis Exp 75:e50330.
Toon S (1996) The relevance of pharmacokinetics in the development of biotechnology products. Eur J Drug Metab Pharmacokinet 21:93-103.

Vasquez KO, Casavant C, and Peterson JD (2011) Quantitative whole body biodistribution of fluorescent-labeled agents by non-invasive tomographic imaging. PLoS One 6:e20594.

Vasquez KO and Peterson JD (2017) Early detection of acute drug-induced liver injury in mice by noninvasive near-infrared fluorescence imaging. $J$ Pharmaco Exp Ther 361:87-98.

Zhang J, Preda DV, Vasquez KO, Morin J, Delaney J, Bao B, Percival MD, Xu D, McKay D, Klimas M, et al. (2012) A fluorogenic near-infrared imaging agent for quantifying plasma and local tissue renin activity in vivo and ex vivo. Am J Physiol Renal Physiol 303:F593-F603.

Zhang Q, Tomazela D, Vasicek LA, Spellman DS, Beaumont M, Shyong B, Kenny J, Fauty S, Fillgrove K, Harrelson J, et al. (2016) Automated DBS microsampling, microscale automation and microflow LC-MS for therapeutic protein PK. Bioanalysis 8:649-659.

Address correspondence to: Dr. Jeffrey D. Peterson, PerkinElmer Discovery \& Analytical Sciences, 68 Elm St., Hopkinton, MA 01748. E-mail: jeff.peterson@perkinelmer.com 